\title{
Current diagnosis and management of late complications in adult congenital heart disease
}

Improvements in quality and level of care in pediatric and adult cardiology, cardiovascular surgery, intensive care as well as improvements in pharmacotherapy resulted in the survival and increased life expectancy of congenital heart disease (CHD) patients to adulthood. At present, in the industrialized world, up to $97 \%$ of children born with CHD reach adulthood. Surgical treatment made survival to adulthood possible for CHD patients; however, these operations are seldom curative. Postoperative residua, sequellae, new onset long-term surgical complications and cardiac and non-cardiac comorbidities adversely impair the long-term course of CHDs and, therefore, require regular medical follow-up by adult congenital heart disease (ACHD) specialists or cardiologists experienced in the care of these patients. Particularly patients with moderate to complex lesions, both unoperated and postoperative, require lifelong surveillance. Because of this, a number of specialized ACHD centers have emerged in the past 3-4 decades in Europe and North America, followed by the Asia-Pacific region, to respond to this need. This evolving field has been developing day by day, and in 2015, examination for board certification as one of the internal medicine subspecialties was initiated in the North America and possibly the trend developed internationally in Europe and Japan. In Germany, there is a special situation, since certification for ACHD specialists (pediatric cardiologists or cardiologists in practice, clinics and centers) has been in place for several years by national medical societies.

Residua and sequelae may progress in severity with age and induce late complications, such as arrhythmias, cardiac failure, thromboembolism, aortopathy, and pulmonary hypertension and infective endocarditis. Prophylaxis and medical and invasive management such as reoperation, cardiac intervention and arrhythmia ablation are mandatory for ACHD patients. There are other issues that further complicate ACHD, including pregnancy and delivery, non-cardiac surgery, hepatitis, metabolic syndrome, psychosocial problems, rehabilitation, health insurance, and extra-cardiac complications. Proper prophylaxis and management of late complications are crucial. During close follow-up process, non-invasive examination especially echocardiography, CT and MRI, arrhythmia monitoring or cardiopulmonary exercise testing for evaluation of these late complications is necessary.

Health promotion and salutogenesis provide a new complementary scientific and practical approach to medicine, which may help to create, maintain and restore wellness in ACHD.

To this end, it is necessary to establish a medical network of care system including internists, local care centers, and specialized ACHD care centers and their multidisciplinary teams. Proper transition from pediatric cardiologists and cardiovascular surgeons to adult CHD caring team, including adult CHD specialists and/or cardiologists trained in this field, is mandatory. A seamless care system for CHD patients from childhood to adulthood can be promoted with excellent care of ACHD patients in the near future. Also, it is important to propagate that during seamless follow up process, patients should visit these specialized centers for keeping on proper follow-up process.

This 3rd focused series on ACHD in CDT provides the current knowledge of imaging such as echocardiography and MRI for evaluation of pathophysiology of aortopathy, cardiac failure, arrhythmias, infective endocarditis, anticoagulation management, medical rehabilitation, QOL and mental problems, metabolic syndrome, liver disease, and salutogenesis in the ACHD field. Also, there are timely narrative reviews on rare disorders such as Ebstein disease and unicuspid aortic valve, and health promotion and salutogenesis. Also, there is an issue on current situation of health care on ACHD patients. These contributions will help understanding future proper management of ACHD.

\section{Acknowledgments}

We would like to thank the CDT-Journal and especially Prof. Paul Schoenhagen, editor in chief, for their dedication to the field of ACHD and for giving this topic space for scientific publications. We are already looking forward to working with them on the conception of a fourth volume soon.

Funding: None. 


\section{Footnote}

Conflicts of Interest: All authors have completed the ICMJE uniform disclosure form (available at http://dx.doi.org/10.21037/ cdt-21-165). The series "Current Management Aspects in Adult Congenital Heart Disease (ACHD): Part III" was commissioned by the editorial office without any funding or sponsorship. KN serves as an unpaid editorial board member of Cardiovascular Diagnosis and Therapy from Oct 2018 to Sep 2022. HK serves as an unpaid editorial board member of Cardiovascular Diagnosis and Therapy from Feb 2018 to Jan 2022. YVK serves as an unpaid editorial board member of Cardiovascular Diagnosis and Therapy from Oct 2018 to Sep 2022. All authors served as the unpaid Guest Editors of the series. The authors have no other conflicts of interest to declare.

Provenance and Peer Review: This article was commissioned by the editorial office, Cardiovascular Diagnosis and Therapy for the series "Current Management Aspects in Adult Congenital Heart Disease (ACHD): Part III". The article did not undergone external peer review.

Ethical Statement: The authors are accountable for all aspects of the work in ensuring that questions related to the accuracy or integrity of any part of the work are appropriately investigated and resolved.

Open Access Statement: This is an Open Access article distributed in accordance with the Creative Commons AttributionNonCommercial-NoDerivs 4.0 International License (CC BY-NC-ND 4.0), which permits the non-commercial replication and distribution of the article with the strict proviso that no changes or edits are made and the original work is properly cited (including links to both the formal publication through the relevant DOI and the license). See: https://creativecommons.org/licenses/by-nc$\mathrm{nd} / 4.0 /$.

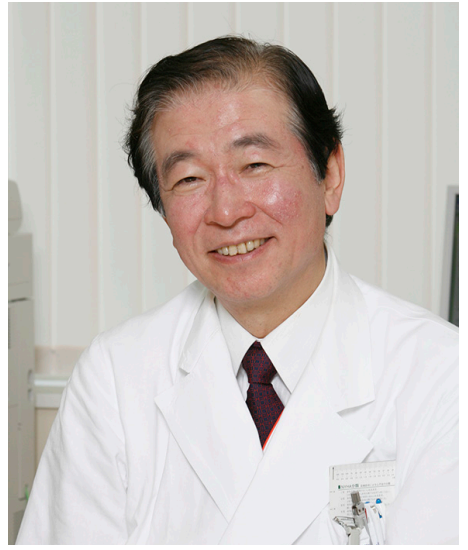

Koichiro Niwa

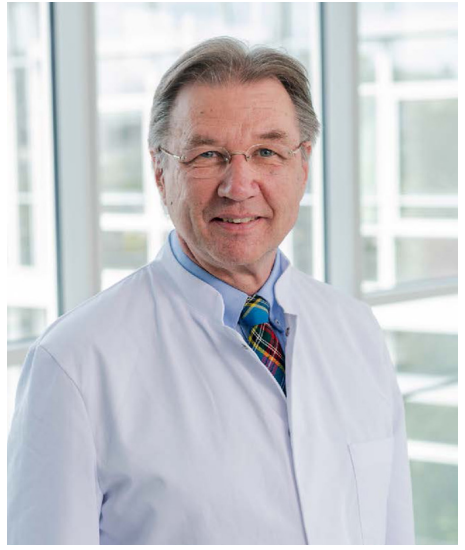

Harald Kaemmerer

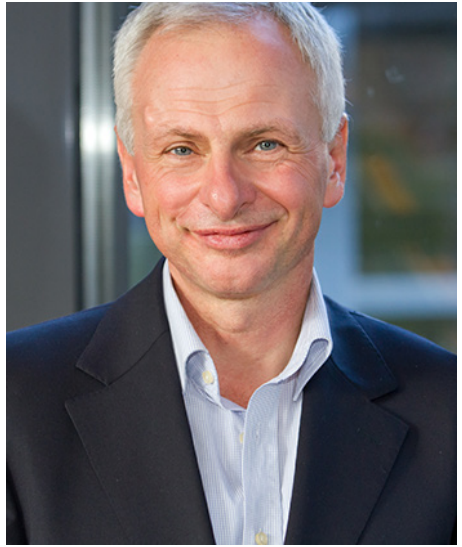

Yskert von Kodolitsch 
Koichiro Niwa, MD, PhD

Executive Advisor, Cardiovascular Center, St. Luke’s International Hospital, Tokyo, Japan. (Email: kniwa@aol.com)

Harald Kaemmerer, MD, VMD

Professor of Internal Medicine/Cardiology, Department of Congenital Heart Disease and Pediatric Cardiology,

German Heart Center Munich, Technical University Munich, Munich, Germany. (Email: Kaemmerer@dbm.mbn.de)

Yskert von Kodolitsch, MD, MBA

Clinic of Cardiology, University Clinic Hamburg-Eppendorf, Hamburg, Germany. (Email: kodolitsch@uke.de)

Submitted Mar 18, 2021. Accepted for publication Mar 31, 2021.

doi: $10.21037 / \mathrm{cdt}-21-165$

View this article at: http://dx.doi.org/10.21037/cdt-21-165

Cite this article as: Niwa K, Kaemmerer $\mathrm{H}$, von Kodolitsch

Y. Current diagnosis and management of late complications

in adult congenital heart disease. Cardiovasc Diagn Ther

2021;11(2):478-480. doi: 10.21037/cdt-21-165 